\title{
The CLIC Physics Potential
}

\author{
Aidan Robson* ${ }^{* \dagger}$ \\ University of Glasgow, G12 8QQ, UK \\ E-mail: aidan.robsoneglasgow.ac.uk
}

\begin{abstract}
The physics and detector studies for the Compact Linear Collider (CLIC) are introduced. A staged programme of $\mathrm{e}^{+} \mathrm{e}^{-}$collisions covering $\sqrt{s}=380 \mathrm{GeV}, 1.5 \mathrm{TeV}$, and $3 \mathrm{TeV}$ would allow precise measurements of Higgs boson couplings, in many cases to the percent level. This corresponds to precision higher than that expected for the high-luminosity Large Hadron Collider. Such precise Higgs coupling measurements would allow sensitivity to a variety of new physics models and the ability to distinguish between them. In addition, new particles directly produced in pairs could be measured with great precision, and measurements in the top-quark sector would provide sensitivity to new physics effects at the scales of tens of $\mathrm{TeV}$.
\end{abstract}

XXIV International Workshop on Deep-Inelastic Scattering and Related Subjects

11-15 April, 2016

DESY Hamburg, Germany

\footnotetext{
*Speaker.

†n behalf of the CLICdp collaboration.
} 


\section{CLIC Overview}

The Compact Linear Collider, CLIC, is a future high-energy $\mathrm{e}^{+} \mathrm{e}^{-}$collider project under development at CERN. A staged programme is proposed, with baseline energies of $\sqrt{s}=380 \mathrm{GeV}$, 1.5 TeV, and $3 \mathrm{TeV}$. The low-energy stage targets precision Standard Model physics focusing on Higgs boson and top-quark measurements, and the higher-energy stages extend that programme and increase the sensitivity to a wide range of beyond-Standard Model (BSM) effects.

In order to realise a multi-TeV collider with a relatively small size, CLIC is designed to have an accelerating gradient of up to $100 \mathrm{MV} / \mathrm{m}$. A $3 \mathrm{TeV}$ collider would therefore be achievable in a complex that is $50 \mathrm{~km}$ long. In order to reach such a high accelerating gradient, the collider is based on room-temperature accelerating structures and a two-beam acceleration scheme, where power from a low-energy, high-current drive beam is extracted and used to accelerate the main beam.

The CLIC/CTF3 collaboration, consisting of 62 institutes from 28 countries, works on the design and development of the accelerator and the construction and operation of the CLIC Test Facility (CTF3) at CERN. Experiments at CTF3 have demonstrated the feasibility of the drivebeam generation and the two-beam acceleration scheme, with a gradient up to $145 \mathrm{MV} / \mathrm{m}$.

The CLIC Detector and Physics (CLICdp) collaboration, involving 27 institutes from 17 countries, focuses on the physics prospects of the collider, detector optimisation, and detector R\&D with the aim of providing demonstrators for the main technical challenges.

The CLIC Conceptual Design Report (CDR) was completed in 2012 [1] and the collaboration is currently developing a Project Plan for the next European Strategy update. Construction of CLIC, once approved, could start in the mid-2020s and lead to physics at the first energy stage as soon as around 2035.

\section{CLIC Detector}

The CLIC detector design is determined by the physics performance requirements in the demanding environment resulting from the bunch structure of the beams. The main beams consist of bunch trains $156 \mathrm{~ns}$ long with $0.5 \mathrm{~ns}$ bunch spacing within the trains, and the trains are separated by $20 \mathrm{~ms}$. High bunch-charge density leads to significant beamstrahlung and beam-beam effects of incoherent $\mathrm{e}^{+} \mathrm{e}^{-}$pair production and hadron production in $\gamma \gamma$ collisions. This results in a high detector occupancy and a need for precise timing, in particular a nanosecond time-stamping in the vertex detector. The expected tracking resolution is $\sigma\left(p_{T}\right) / p_{T}^{2} \sim 2 \times 10^{-5} \mathrm{GeV}^{-1}$, to allow for example precise measurement of the Higgs boson recoil from $\mathrm{HZ}$ events with $\mathrm{Z} \rightarrow \mu \mu$, and for measurement of the rare decay $\mathrm{H} \rightarrow \mu \mu$. The assumed calorimeter resolution is $\sigma(E) / E \sim 3.5 \%$ for $E>100 \mathrm{GeV}$, which should allow the decays of $\mathrm{W}$ and $\mathrm{Z}$ bosons to hadrons to be distinguished; and the calorimeters need to have very fine granularity in order to allow for a particle flow reconstruction approach [2] to be fully effective.

Initial studies of the CLIC physics potential used variants of the ILD and SiD detector designs for the International Linear Collider (ILC), adapted for the CLIC environment. The experience from those studies is currently being used to define a single, optimised detector concept for CLIC. The main features of the detector are: an ultra low-mass vertex detector with $25 \mu \mathrm{m}$ pixels; a 
silicon tracker with large pixels or strips having readout granularity approximately $50 \mu \mathrm{m} \times(1-$ 10) $\mathrm{mm}$ and material budget of $1-2 \% X_{0}$ per layer; a SiW electromagnetic calorimeter with cell size of $5 \times 5 \mathrm{~mm}^{2}$ and a steel-absorber hadronic calorimeter with cell size of $30 \times 30 \mathrm{~mm}^{2}$; all surrounded by a $4 \mathrm{~T}$ solenoid. The solenoid return yoke is instrumented with muon detectors, and the forward region is instrumented with detectors dedicated to the luminosity measurement and the beam monitoring (LumiCal and BeamCal). The calorimeters are developed in cooperation with the CALICE collaboration, and the LumiCal and BeamCal in cooperation with the FCAL collaboration.

\section{Physics Highlights}

The three pillars of the CLIC physics programme are Higgs boson physics, top-quark physics, and beyond-Standard Model (BSM) physics. Some recent developments in each of these areas are discussed in the following sections.

\subsection{Higgs physics}

While WW fusion is the dominant Higgs production mechanism in $\mathrm{e}^{+} \mathrm{e}^{-}$collisions above $\sqrt{s} \sim 450 \mathrm{GeV}$, Higgs-strahlung (HZ production) will dominate at the energy of the first CLIC stage. As the initial state is known and provides a kinematic constraint, the total Higgs-strahlung cross-section $\sigma(\mathrm{HZ})$ can be measured without reconstructing the Higgs decay, by looking only at the decay of the $\mathrm{Z}$ boson; this is the recoil mass method. Knowing $\sigma(\mathrm{HZ})$ allows measurements of all other Higgs cross-sections times branching ratios to be converted to Higgs branching ratios. The total cross-section $\sigma(\mathrm{HZ})$ is proportional to the square of the $\mathrm{H}-\mathrm{Z}$ coupling, $g_{\mathrm{HZZ}}^{2}$. The measurement of $\sigma(\mathrm{HZ}) \cdot \operatorname{Br}(\mathrm{H} \rightarrow \mathrm{ZZ})$, which is proportional to $g_{\mathrm{HZZ}}^{4} / \Gamma_{\mathrm{H}}$, thus allows the extraction of the Higgs boson width $\Gamma_{\mathrm{H}}$. Determination of $\Gamma_{\mathrm{H}}$ allows all the Higgs couplings to be extracted from the branching fractions. Unlike at the LHC, all final states (e.g. $\mathrm{H} \rightarrow \mathrm{cc}$ ) can be measured directly, $\Gamma_{\mathrm{H}}$ is a free parameter in the final fit, and no assumptions are needed about invisible Higgs decays, and so the coupling measurements are 'model-independent'.

A comprehensive set of Higgs studies for CLIC has recently been completed, incorporating more than 20 individual analyses. Highlights include the new incorporation of hadronic $\mathrm{Z}$ decays into the Higgs recoil method [3], which significantly improves the statistical power of the $g_{\mathrm{HZZ}}$ coupling determination to $0.8 \%$; this is important because, as explained above, it is needed in the extraction of all the other couplings. The separation of $\mathrm{H} \rightarrow \mathrm{bb}, \mathrm{H} \rightarrow \mathrm{cc}$, and $\mathrm{H} \rightarrow \mathrm{gg}$, which relies on the excellent detector performance, has been demonstrated. The Higgs self-coupling should be measurable to $12 \%$ from the high-energy programme (assuming $80 \%$ electron polarisation). Each energy stage is found to contribute significantly to the Higgs programme; the first stage provides the crucial model-independent $\mathrm{Z}$ coupling measurement, and couplings to most fermions and bosons, while the higher-energy stages improve them and add the top-quark, muon, and photon couplings. Adding LHC-like model-dependent constraints for a direct comparison demonstrates precision significantly better than the high-luminosity LHC, for most of the Higgs couplings. The significance of precise measurements of the Higgs couplings is their sensitivity to BSM effects, which will be discussed below. A summary of the expected precision is given in Figure 1 and a dedicated paper is forthcoming. The Higgs mass measurement has also been studied, and the 
projected sensitivity at the end of the high-energy run, and assuming a polarised electron beam, is $24 \mathrm{MeV}$, which compares very favourably with the HL-LHC projection of $50 \mathrm{MeV}$ [4].

\subsection{Top-quark physics}

The top quark has so far been observed only in hadronic collisions, and an $\mathrm{e}^{+} \mathrm{e}^{-}$collider running at and above the t⿱t threshold will allow many valuable measurements. At CLIC, a threshold scan around $\sqrt{s}=350 \mathrm{GeV}$ is foreseen during the low-energy running period, encompassing ten points and taking around one year. The shape of the $t \bar{t}$ cross-section around threshold is very sensitive to the top-quark mass, width, and couplings, and the threshold scan will allow extraction of the $1 S$ mass, which is theoretically well-defined, with a precision of around $50 \mathrm{MeV}$ [5]. The shape of the cross-section, dependence on mass and expected statistical uncertainties are shown in Figure 1. Measurements of top-quark asymmetries can be sensitive to BSM effects, and this is discussed below.
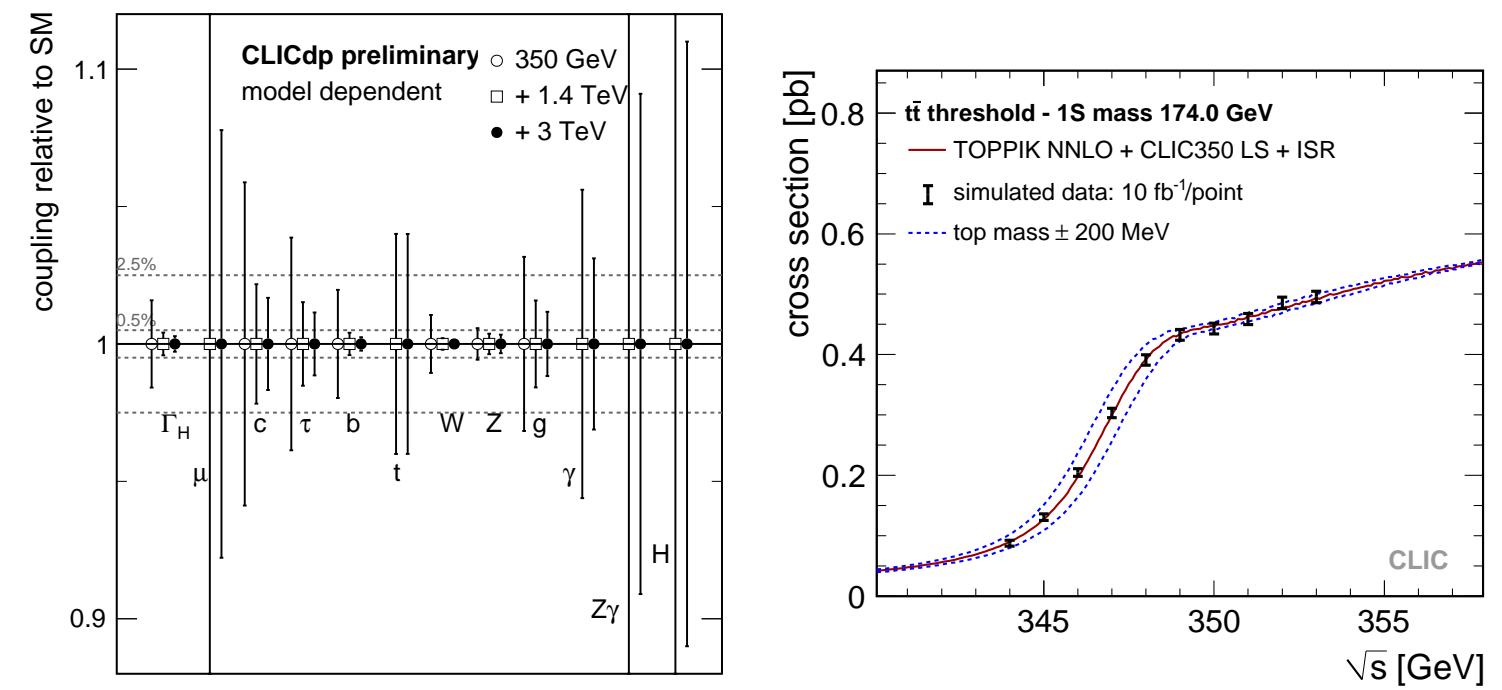

Figure 1: (left) Model-dependent Higgs coupling sensitivities for the three CLIC energy stages; (right) Background-subtracted simulated $t \bar{t}$ cross-section measurement showing the effect on the theoretical prediction of a $\pm 200 \mathrm{MeV}$ mass shift (plot from [5]).

\subsection{BSM physics}

The Higgs programme will result in coupling measurements with precision in many cases of the order of $1 \%$, as shown in Figure 1. BSM effects can modify the effective Higgs couplings from their Standard Model values, and the expected pattern of these deviations (coupling to vector bosons, third-generation fermions, and effective photon couplings) is different in different models. For example in scenarios with new particles at a mass scale of $1 \mathrm{TeV}$, a two-Higgs-doublet model could enhance the $\mathrm{H}-\mathrm{b}$ coupling by around $10 \%$ while only increasing the vector and photon couplings by $1 \%$, whereas a composite Higgs model could suppress all the couplings by up to $9 \%$ [4]. The precise Higgs coupling measurements therefore allow any deviations to 'fingerprint' the underlying physics origins. 
In the top-quark sector, the general $\mathrm{ttX}$ vertex can be parameterised using form factors for Standard Model and BSM terms, which can be related to the left- and right-handed couplings of the top quark to the $\mathrm{Z}$ boson [6]. As with the Higgs couplings, those left- and right-handed couplings can be modified from their Standard Model values under different BSM scenarios, and the precise measurements possible at CLIC would allow a 'fingerprinting' of any underlying effect. The top coupling measurements at the ILC at $\sqrt{s}=500 \mathrm{GeV}$ have been shown to be sensitive to scales of $\sim 25 \mathrm{TeV}$ in typical scenarios; the CLIC analysis is ongoing, but the impact of four-fermion operators increases strongly with $\sqrt{s}$ to CLIC energies.

New particles that have mass below $\sqrt{s} / 2$ could be pair-produced at CLIC. Their properties, including mass, spin, and quantum numbers, can be precisely measured with the help of polarised beams. The excellent detector resolution will ensure that particle masses can be determined even in the difficult cases, for example when chargino and neutralino masses are similar; in that case their decays to $\mathrm{W}, \mathrm{Z}$ and Higgs bosons can be distinguished in a two-dimensional invariant mass plane and masses measured to a few percent [7]. Similarly, even when charged and neutral supersymmetric Higgs particles have similar masses, they can be distinguished by the excellent reconstruction of their b-quark decays [1].

\section{Outlook}

The CLIC accelerator is in an advanced state of development, and the detector concept is mature. The first energy stage will provide precise measurements of many Higgs boson couplings, which will be improved by the subsequent high-energy running; and a set of comprehensive simulation studies of the Higgs physics sensitivity is complete. High-energy running will provide significant discovery potential for BSM phenomena. Accelerator and physics studies are ongoing, in preparation for the next European Strategy for Particle Physics update.

\section{References}

[1] M. Aicheler et al., A Multi-TeV Linear Collider based on CLIC Technology, CLIC Conceptual Design Report, [CERN-2012-007]; L. Linssen et al., Physics and Detectors at CLIC: CLIC Conceptual Design Report, [CERN-2012-003].

[2] M. A. Thomson, Particle Flow Calorimetry and the PandoraPFA Algorithm, Nucl. Instrum. Methods, A611 (2009) 25 [arXiv:0907.3577].

[3] M. A. Thomson, Model-independent measurement of the $e^{+} e \rightarrow H Z$ cross section at a future $e^{+} e$ linear collider using hadronic Z decays, Eur. Phys. J. C76 (2016) 72 [arXiv: 1509 . 02853].

[4] S. Dawson et al., Higgs Working Group Report of the Snowmass 2013 Community Planning Study, arXiv: 1310.8361.

[5] K. Seidel, F. Simon, M. Tesar, and S. Poss, Top quark mass measurements at and above threshold at CLIC, Eur. Phys. J. C 73 (2013) 2530 [arXiv:1303.3758].

[6] M. Amjad et al., A precise characterisation of the top quark electro-weak vertices at the ILC, Eur. Phys. J. C (2015) 75:512 [arXiv:1505.06020].

[7] T. Barklow, A. Munnich, and P. Roloff, Measurement of chargino and neutralino pair production at CLIC, CERN LCD-Note-2011-037. 